02

\title{
Оптико-энергетические свойства тонких пленок CdS, полученных методом высокочастотного магнетронного распыления
}

\author{
(C) Р.Ю. Петрусь ${ }^{1}$, Г.А. Ильчук ${ }^{1, \uparrow}$, А.И. Кашуба ${ }^{1,2}$, И.В. Семкив ${ }^{1}$, Е.О. Змийовська ${ }^{1}$ \\ ${ }^{1}$ Национальный университет „Львовская политехника“, \\ 79013 Львов, Украина \\ 2 Львовский национальный университет имени Ивана Франко, \\ 79005 Львов, Украина \\ ๑e-mail: ilchukg@gmail.com
}

Поступила в редакцию 13.11.2018 г.

В окончательной редакции 13.11.2018 г.

Принята к публикации 20.11.2018 г.

\begin{abstract}
Представлены результаты экспериментальных и теоретических исследований оптико-энергетических свойств тонких пленок CdS. Приведены методика синтеза, результаты структурных и оптических исследований тонких пленок $\mathrm{CdS}$, осажденных на поверхности стеклянной подложки. В рамках метода псевдопотенциала теоретически изучена динамика изменения параметров электронной подсистемы пленки $\mathrm{CdS}$. Установлены прямозонный характер запрещенной щели пленки и генезис зоны проводимости и валентной зоны. С использованием соотношения Крамерса-Кронига получены спектры поглощения и отражения, которые удовлетворительно коррелируют с полученными экспериментальными данными.
\end{abstract}

DOI: $10.21883 /$ OS.2019.03.47370.327-18

\section{Введение}

Полупроводниковое соединение $\mathrm{CdTe}$ зарекомендовало себя как одно из ведущих для изготовления экономически выгодных фотоэлектрических устройств второго поколения. Солнечные батареи на основе CdTe привлекают внимание, поскольку CdTe характеризуется прямой запрещенной зоной шириной $1.46 \mathrm{eV}$ и высоким коэффициентом поглощения (выше $10^{5} \mathrm{~cm}^{-1}$ ) [1], что делает его отличным поглощающим слоем солнечных элементов.

Для формирования гетеропереходов высокой эффективности на основе $p$-СdТе для оконного слоя солнечной батареи в основном используется кадмий сульфид $(\mathrm{CdS})[2-4] . \mathrm{CdS}$ характеризуется высоким коэффициентом поглощения и высокой фотопроводностью в видимой области. Это связано с тем, что $\mathrm{CdS}$ является прямозонным полупроводником и характеризуется соответственно прямыми оптическими переходами зона-зона. Ширина запрещенной зоны при комнатной температуре составляет $2.42 \mathrm{eV}$, а коэффициент преломления - 2.52 . Электрические свойства $\mathrm{CdS}$ характеризуются удельным сопротивлением порядка $10^{6} \mathrm{Om} \cdot \mathrm{cm}$ и $n$-типом проводимости.

$\mathrm{CdS}$ характеризуется кубической и гексагональной фазами, зависящими главным образом от выбора методов синтеза и параметров роста [5-8]. В солнечных элементах на основе гетерострукы CdS/CdTe толщина $\mathrm{CdS}$ в большинстве случаев составляет около 150-300 nm [9]. Фотогенерированные носители заряда в пленке $\mathrm{CdS}$ практически полностью рекомбинируют внутри и не создают фототок. Из-за поглощения света пленкой $\mathrm{CdS}$ фототок в структуре не возникает. Поэтому для изготовления высокоэффективных солнечных элементов на основе $\mathrm{CdTe}$ желательно использовать пленку $\mathrm{CdS}$ толщиной менее $100 \mathrm{~nm}[9,10]$.

Перечисленные свойства тонкопленочных соединений на основе $\mathrm{CdS}$ обеспечивают актуальность дальнейших исследований. Именно поэтому целью работы являются теоретические и экспериментальные исследования оптических и энергетических свойств тонких пленок $\mathrm{CdS}$.

\section{Экспериментальная часть}

\section{Осаждения тонких пленок CdS методом высокочастотного магнетронного напыления}

Пленки CdS осаждены на стеклянные подложки и подложки стекло/ITO (NANOCS IT100-111-25, 100 Ohm/sq) размером $16 \times 8 \times 1.1 \mathrm{~mm}$ методом высокочастотного (ВЧ) магнетронного напыления $(13.6 \mathrm{MHz})$ с использованием ВУП-5М (Selmi) [1]. В качестве мишени использовали монокристаллический диск чистоты $99.999 \%$, толщиной 1 и диаметром $40 \mathrm{~mm}$. Расстояние мишень-подложка составляло $60 \mathrm{~mm}$. Продолжительность осаждения составляла $0.25 \mathrm{~h}$, а начало и завершение процесса контролировали с помощью подвижной заслонки.

Перед началом напыления проведено вакуумирование камеры. Давление газов в рабочей камере составляло $4 \cdot 10^{-4} \mathrm{~Pa}$. Это возможно с использованием в паромасляном насосе диффузионной жидкости „Полифениловий 
Эфир 5Ф4Э“, что обеспечивает низкое значение парциального давления $\left(9 \cdot 10^{-7} \mathrm{~Pa}\right)$.

Распыление проводили при давлении аргона (Ar) 1.0-1.3 Ра. Мощность ВЧ магнетрона поддерживали на уровне $30 \mathrm{~W}$, а температуру подложки - при температуpe $573 \mathrm{~K}$. Для нагрева подложек использовали вольфрамовый высокотемпературный нагреватель мощностью $300 \mathrm{~W}$. Контроль температуры осуществляли с помощью ПИД-регулятора для контроля скоростей нагрева и охлаждения, а также для обеспечения температурного режима осаждения. Толщину пленок измеряли на профилометре Veeco, модель Dektak 8. Также проводили расчеты по методике [11], основанной на определении интерференционных максимумов и минимумов в спектральной зависимости коэффициента пропускания. Результаты расчета хорошо согласуется с экспериментально определенными значениями толщины пленки. Установлено, что средняя скорость осаждения пленок составляла $3.9-4.2 \AA / \mathrm{s}$.

\section{Методы структурных исследований}

Массивы экспериментальных интенсивностей и углов отражения от исследуемых образцов получены на дифрактометре STOE STADI P с линейным позиционнопрецизионным детектором PSD по схеме геометрии Брегга-Брентано на отражение $\left(\mathrm{Cu} K_{\alpha 1}\right.$-излучение; во-

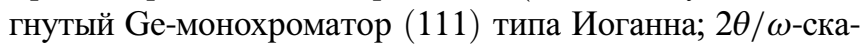
нирование, интервал углов $20.000^{\circ} \leq 2 \theta \leq 117.905^{\circ}$ с шагом $0.015^{\circ}$, температура комнатная). Обработку экспериментальных дифракционных массивов, расчет теоретических дифрактограмм, рентгеновский фазовый анализ, индексирование параметров элементарных ячеек проводили с помощью пакета программ STOE WinXPOW и программы PowderCell [12].

Морфологию поверхности полученных пленок $\mathrm{CdS}$ исследовали с использованием сканирующего электронного микроскопа JSM 6700F.

\section{Методика расчета}

Теоретические расчеты проведены в рамках теории функционала электронной плотности (DFT).

Для расчета свойств монокристаллического $\mathrm{CdS}$ использовали кристаллическую решетку, основные параметры которой приведены ниже. Для ионных потенциалов использовались ультрамягкие псевдопотенциалы Вандербильта [13]. Для описания обменнокорреляционной энергии электронной подсистемы использовался функционал в приближении обобщенного градиента (GGA) в параметризации Пердью, Бурке и Ернцергофа (PBE) [14]. Для корреляционного потенциала к границам высокой плотности применены формула Кеперли-Алдера и соотношение Гелл-Мана-Бракнера.

Распределение зарядовой плотности вычисляли методом специальных точек с привлечением техники демпфирования заряда. Для каждой кристаллической струк- туры проводили релаксации позиций ионов на основе рассчитанных атомных сил и определяли интегральное напряжение ячейки.

Для расчетов использовали значение энергии обрезания плоских волн $E_{\text {cut-off }}=290 \mathrm{eV}$. Сходимость общей энергии составляла около $5 \cdot 10^{-6} \mathrm{eV} /$ atom. Электронная конфигурация для атомов такова: $\mathrm{Cd}-[\mathrm{Kr}] 4 d^{10} 5 s^{2}$, $\mathrm{S}-[\mathrm{Ne}] 3 s^{2} 3 p^{4}$, где состояния $[\mathrm{Kr}]$ и $[\mathrm{Ne}]$ принадлежат к остову. Интегрирование по зоне Бриллюэна выполнялось по сетке $2 \times 2 \times 2 k$-точек с помощью схемы Монхорста-Пака [15]. Спиновые степени свободы электронов в этом расчете не учтены [16].

Оптимизация атомных координат и параметров элементарной ячейки проведено с использованием метода Бройдена-Флетчера-Гольдфарба-Шани (BFGS) [17]. Оптимизацию продолжали до тех пор, пока силы, действующие на атомы, не стали меньше $0.01 \mathrm{eV} / \AA$, максимальное смещение $<10^{-6} \AA$, а механические напряжения в ячейке $<0.02 \mathrm{GPa}$. Зонно-энергетическую диаграмму построили по точкам зоны Бриллюэна в обратном пространстве: $\Gamma(0,0,0), \mathrm{A}(0,0,0.5), \mathrm{H}(-0.333,0.667,0.5)$, $\mathrm{K}(-0.333,0.667,0), \mathrm{M}(0 ; 0.5 ; 0), \mathrm{L}(0 ; 0.5 ; 0.5)$ для кристалла и $\Gamma(0,0,0), \mathrm{A}(0,0,0.5), \mathrm{H}(-0.333,0.667,0.5)$, $\mathrm{K}(-0.333,0.667,0)$ для пленки.

Модели пленки $\mathrm{CdS}$ формировали из объемного кристалла CdS вдоль оси [001]. Расстояние между формульными единицами увеличивалось приблизительно на $15 \mathrm{~nm}$, образуя таким образом вакуумную щель, чтобы избежать взаимодействия между слоями.

Теоретически рассчитана зонно-энергетическая диаграмма тонких пленок толщиной около $10 \mathrm{~nm}$, что меньше, чем в экспериментально синтезированных пленках $\mathrm{CdS}(380 \mathrm{~nm})$. Поскольку ab initio расчеты пленок толщиной $300 \mathrm{~nm}$ требуют значительных компьютерных ресурсов, представленные ниже результаты могут отражать влияние размерного эффекта на свойства пленки.

Известной проблемой данного подхода к расчетам является занижение ширины запрещенной зоны. Наиболее простым способом получить близкие к эксперименту результаты является применение так называемого „оператора ножницы“ („scissors operator"), что приводит к изменению ширины запрещенной щели путем смещения зон проводимости в область более высоких энергий. Это оператор, основанный на близости дисперсионных зависимостей $E(k)$ энергий зон проводимости, которые определяются из решения уравнений Кона-Шема [18]. Сдвиг зон проводимости расчетного энергетического спектра обычно проводят до достижения экспериментального значения минимальной ширины энергетической щели $E_{g}$ кристалла. В настоящей работе для согласования абсолютных значений $E_{g}$ проведена коррекция расчетной величины на значение $\Delta E=0.73 \mathrm{eV}$. Но это не влияет на общую тенденцию электронных и структурных свойств, что подтверждено рядом предыдущих электронных расчетов [19-21]. 


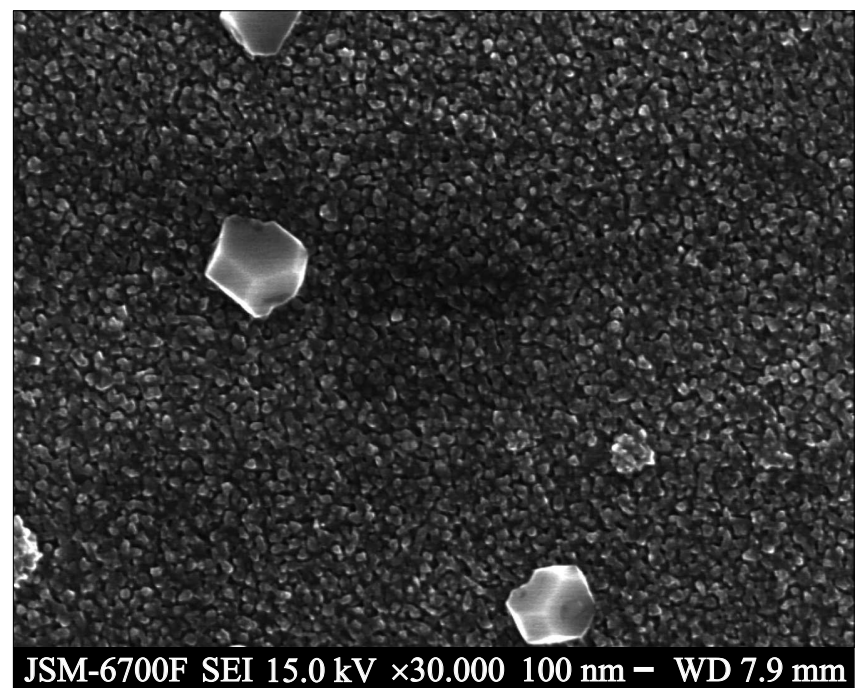

Рис. 1. Морфология поверхности пленки $\mathrm{CdS}$.

Структурные параметры тонких пленок $\mathrm{CdS}$

\begin{tabular}{c|c}
\hline$a, \mathrm{~nm}$ & $0.4048 \pm 0.0016$ \\
\hline$c, \mathrm{~nm}$ & $0.666 \pm 0.004$ \\
\hline$a / c$ & $1.645 \pm 0.011$
\end{tabular}

\section{Результаты и обсуждения}

В процессе магнетронного ВЧ распыления получены пленки $\mathrm{CdS}$ светло-оранжевой окраски, что характерно для соединения $\mathrm{CdS}$. Морфология поверхности полученных пленок $\mathrm{CdS}$ приведена на рис. 1.

Установлено, что подложка равномерно покрыта кристаллитами соединения $\mathrm{CdS}$ с четкой огранкой и средними размерами 30-40 nm. Распределение кристаллитов по размерам показало однородность условий роста по всей плоскости подложки. Заметим, что не столь часто встречаются зерна диаметром около $300 \mathrm{~nm}$.

Из анализа дифрактограммы для $\mathrm{CdS}$ (рис. 2) установлено, что пленка однофазная. Пространственная группа соединения $\mathrm{CdS}-P 6_{3} m c$. Уточненный параметр элементарной ячейки приведен в таблице. Детальный анализ положения экспериментальных рефлексов и их интенсивностей по сравнению с теоретическими пиками показал очень сильную преимущественную ориентацию зерен фазы $\mathrm{CdS}$ в синтезированной пленке (текстуру). Наблюдаются рефлексы от плоскостей с индексами Миллера $h k l(001)$, тогда как рефлексы с другими наборами $h k l$ почти полностью потушены по интенсивности. Присутствующий незначительней фон можно объяснить вкладом подложки.

Проведено исследование спектральной зависимости оптического пропускания (Shimandzu UV-3600) полученных образцов в видимой и ближней инфракрасной областях (рис. 3). Спектры пропускания $T(\lambda)$ пленок
$\mathrm{CdS}$ (рис. 3) характеризуются четко выраженным краем фундаментального поглощения, локализованным в окрестности $500 \mathrm{~nm}$, что является подтверждением наличия соединения кадмия сульфида. Спектры пропускания также характеризуются наличием экстремумов интерференции, что свидетельствует о совершенстве полученных пленок. На рис. 2 видны четкие интерференционные максимумы и минимумы в диапазоне длин волн от 500 до $1500 \mathrm{~nm}$, связанные с набегом фазы на $2 \pi$ в фазовой толщине при изменении длины световой волны. С помощью интерференционной картины оптического пропускания рассчитана толщина пленки $\mathrm{CdS}$ $(h=380 \mathrm{~nm})$.

Спектральная зависимость поглощения пленок $\mathrm{CdS}$ в координатах $(\alpha h v)^{2}-h v$ демонстрирует наличие края фундаментального поглощения (рис. 4).

Проведена экстраполяция линейных участков кривой $(\alpha h v)^{2}$ до пересечения с осью энергий (рис. 4) по методике [22] и определено значение оптической ширины запрещенной зоны пленки $\mathrm{CdS}-2.39 \mathrm{eV}$.

Линейный характер зависимостей $(\alpha h v)^{2}=f(h v)$ в интервале 2.3-2.6 eV указывает на формирование края

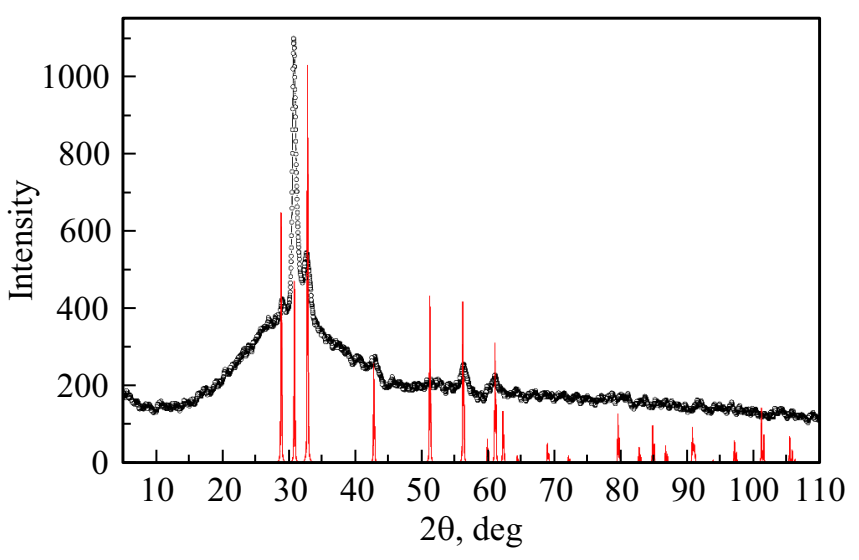

Рис. 2. Экспериментальная дифрактограмма пленки CdS.

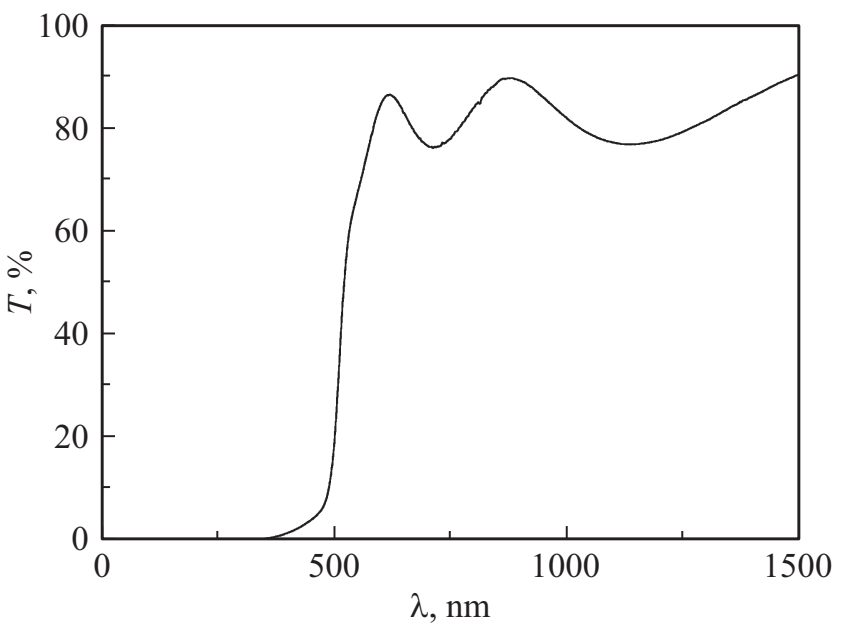

Рис. 3. Спектры пропускания пленки CdS. 


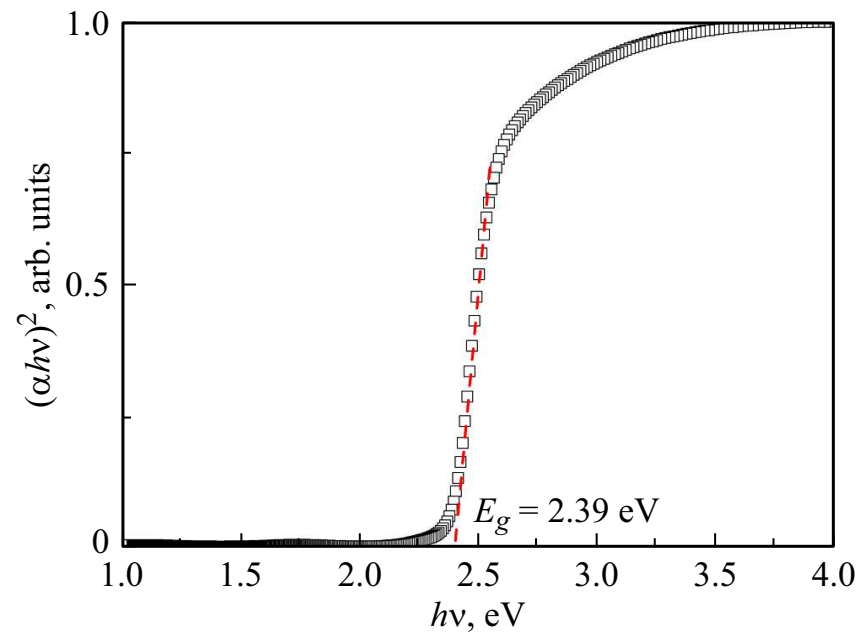

Рис. 4. Спектральная зависимость оптического поглощения для пленки CdS.

поглощения прямыми межзонными оптическими переходами.

На рис. 5 изображена полная зонная энергетическая диаграмма кристалла и тонкой пленки $\mathrm{CdS}$ вдоль высокосимметричних линий тетрагональной зоны Бриллюэна. Здесь энергия отсчитана от уровня Ферми.

Анализ результатов теоретических расчетов зонноэнергетического спектра показывает, что наименьший энергетический промежуток запрещенной зоны локализован в центре зоны Бриллюэна (точка Г). Это значит, что кристалл характеризуются прямой запрещенной зоной. Сравнивая теоретические результаты с литературными экспериментальными данными [23,24], видим, что расчеты в приближениях GGA PBE для кристалла приводят к заниженному значению ширины запрещенной зоны.

Анализ парциальных вкладов отдельных уровней в функцию полной плотности состояний (рис. 6) и парциальных вкладов отдельных зон в электронную плотность позволил определить генезис валентных зон и зоны проводимости кристалла и тонкой пленки $\mathrm{CdS}$. Самые низкие зоны от -12 до $-10 \mathrm{eV}$ сформированы $s$ состояниями S. Формирование следующих зон, диспергированных у энергетической метки от -9 до $-6 \mathrm{eV}$, происходит от вкладов $d$-состояний $\mathrm{Cd}$. Вершина валентного комплекса практически сформирована $p$-состояниями $\mathrm{S}$ c примесью $p$-состояний $\mathrm{Cd}$. Но дно зоны проводимости в основном сформировано из $p$-состояний $\mathrm{Cd}$ и $\mathrm{S}$, а также $s$-состояниями $\mathrm{Cd}$.

Учитывая правила отбора, результаты расчетов зонноэнергетических спектров и плотности состояний, можем предположить, что наименьшую запрещенную щель формируют прямые $s-p$-переходы. Не исключено, что прямозонный переход (локализованный в точке Г зоны Бриллюэна) может быть сформирован соединением $\mathrm{S}-\mathrm{Cd}$.
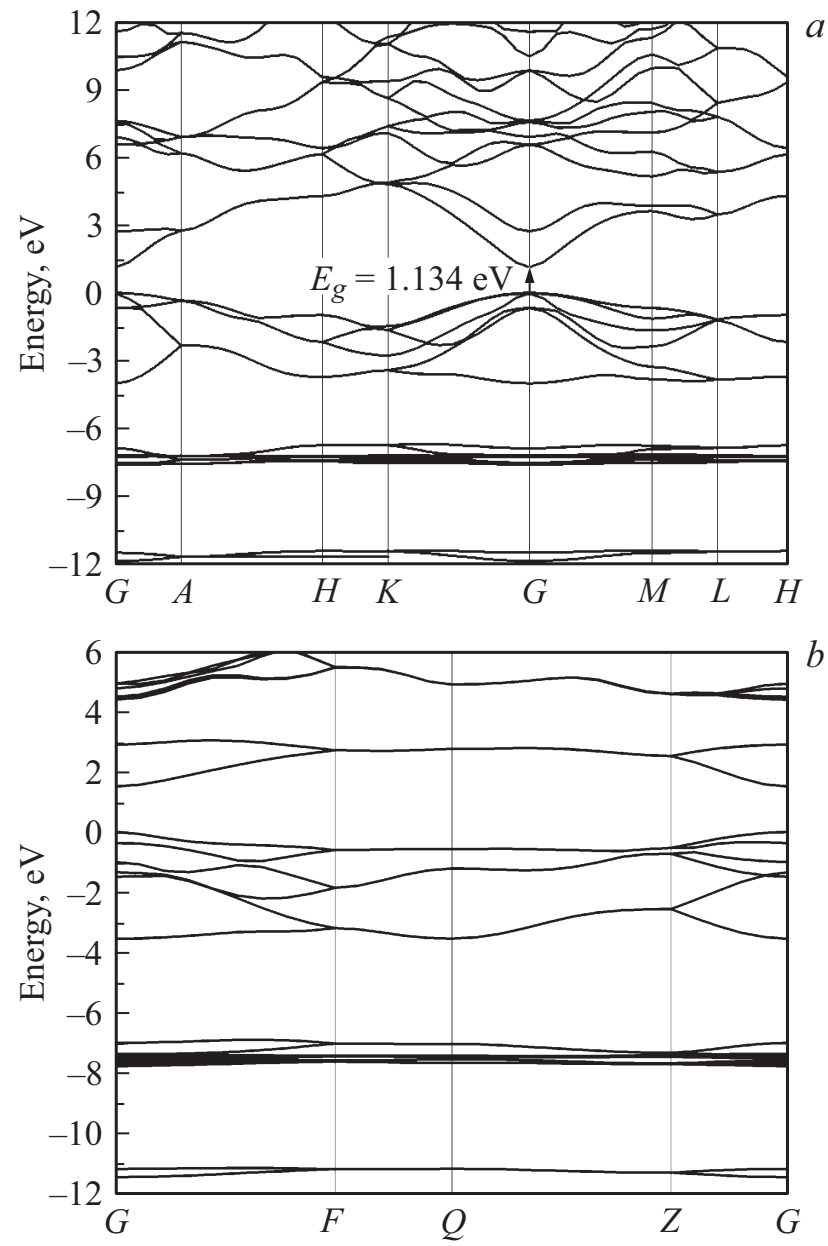

Рис. 5. Зонно-энергетическая диаграмма кристалла $(a)$ и тонкой пленки $(b) \mathrm{CdS}$.

Мнимая часть диэлектрической функции $\varepsilon(\hbar \omega)$ $\left(\varepsilon=\varepsilon_{1}+i \varepsilon_{2}\right)$ в $\mathrm{CdS}$ (рис. 7) рассчитана с использованием следующего соотношения [25]:

$$
\varepsilon_{2}(\hbar \omega)=\frac{2 e^{2} \pi}{V \varepsilon_{0}} \sum_{K, v, c} \mid\left\langle\left.\Psi_{K}^{c}|\hat{\mathbf{u}} \cdot \mathbf{r}| \Psi_{K}^{v}\right|^{2} \delta\left(E_{K}^{c}-E_{K}^{v}-\hbar \omega\right),\right.
$$

где $V$ - объем элементарной ячейки, $E_{K}-$ энергия полосы, индексы $c$ и $v$ относятся к состояниям проводимости и валентной зоны соответственно, $u$ - периодическая часть орбитали ячейки (волновых функций) в $k$-точке.

После этого реальная часть диэлектрической функции $\varepsilon_{1}(\hbar \omega)$ была получена с использованием соотношения Крамерса-Кронига:

$$
\varepsilon_{1}(\hbar \omega)-1=\frac{2}{\pi} \int_{0}^{\infty} \frac{t \varepsilon_{2}(t) d t}{t^{2}-(\hbar \omega)^{2}}
$$

Теоретически рассчитаны спектры оптического поглощения и отражения, представленные на рис. 8. Следует отметить, что все приведенные оптические функции не 
учитывают оператор „ножниц“. Можем наблюдать, что отличие от экспериментальных данных незначительное. То есть можем предположить, что использованную нами методику расчета можно успешно использовать в прогнозировании оптических и энергетических свойств тонких плевок. Исходя из расчетов спектра отражения (рис. 8,a) можем предположить, что пленки $\mathrm{CdS}$ не чувствительны к ультрафиолетовому излучению. Перспективно использование тонких пленок $\mathrm{CdS}$ в качестве оптических окон к солнечным элементам на основе твердых растворов компонентов $\mathrm{A}^{\mathrm{II}} \mathrm{B}^{\mathrm{VI}}$, в том числе таких как $\mathrm{Cd}_{1-x} \mathrm{Mn}_{x} \mathrm{Te}, \mathrm{Cd}_{1-x} \mathrm{Zn}_{x} \mathrm{Te}, \mathrm{Cd}_{1-x} \mathrm{Hg}_{x} \mathrm{Te}$ и $\mathrm{CdTe}_{1-x} \mathrm{~S}_{x}$. Плавное контролируемое изменение ширины запрещенной щели позволит повысить диапазон чувствительности тонких пленок, что особо важно в солнечной энергетике. Возможность применения тонких пленок $\mathrm{CdS}$ в качестве сенсоров различных газов (а не только как материалов к солнечным панелям) должна стать основой дальнейших исследований.

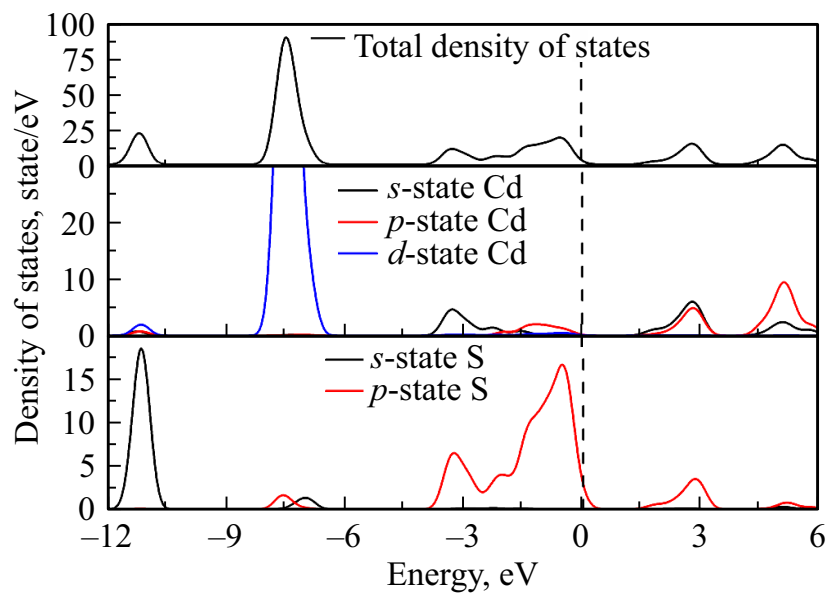

Pис. 6. Плотность состояний тонкой пленки CdS.

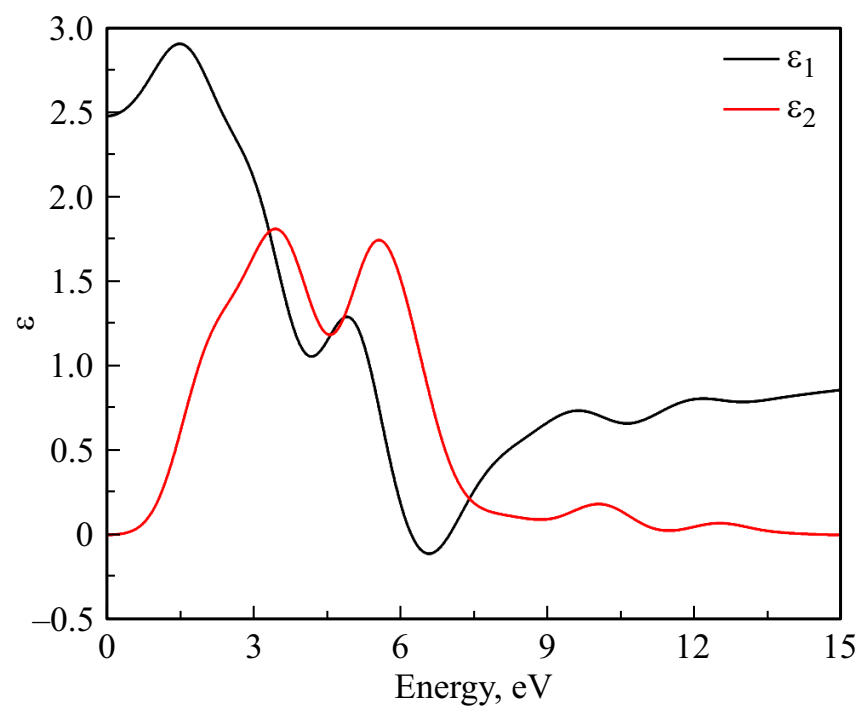

Рис. 7. Действительная и мнимая части функции диэлектрической проницаемости $\varepsilon$ тонкой пленки $\mathrm{CdS}$.
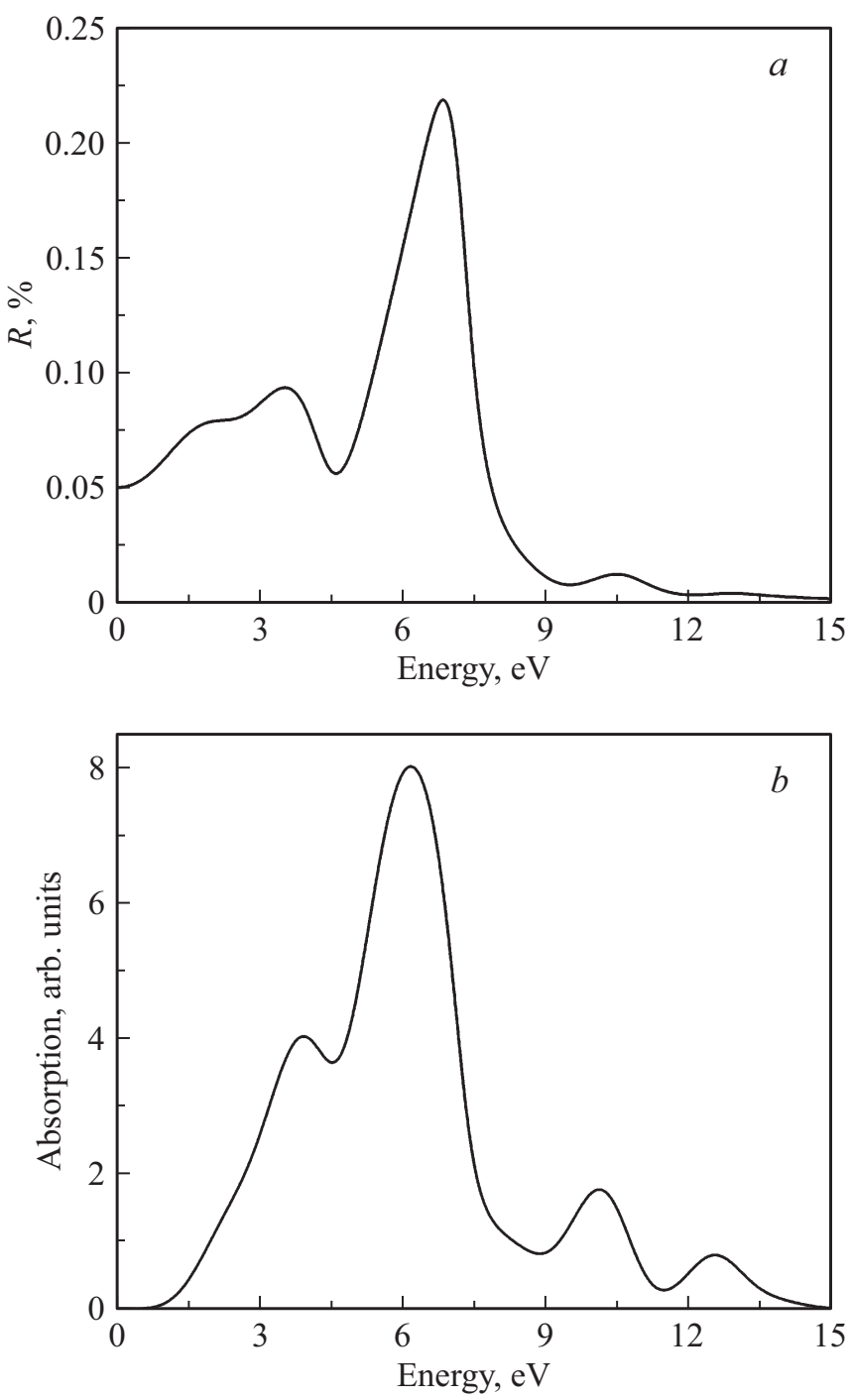

Рис. 8. Теоретически рассчитанные спектры оптического отражения $(a)$ и поглощения $(b)$ тонкой пленки $\mathrm{CdS}$.

\section{Выводы}

Микрофотографии поверхности пленок показали формирование однородных пленок с равномерным распределением зерен соединения. Результаты рентгеноструктурного анализа подтвердили формирования полупроводникового соединения $\mathrm{CdS}$ (пространственная группа $\left.P 6_{3} m c\right)$. Уточнен параметр элементарной гранецентрированной кубической ячейки.

На спектральных зависимостях оптического пропускания наблюдается наличие края фундаментального поглощения, положение которого соответствует значениям, характерным для соединения $\mathrm{CdS}$. На спектре оптического поглощения образца $\mathrm{CdS}$ наблюдается край фундаментального поглощения, на основе которого удалось установить значение оптической ширины запрещенной зоны - $2.39 \mathrm{eV}$.

На основе теоретических расчетов установлено, что монокристаллический образец и тонкая пленка $\mathrm{CdS}$ об- 
ладают прямой запрещенной щелью, локализованной в точке Г зоны Бриллюэна. Установлен генезис зоны проводимости и запрещенной зоны. Значительных различий в плотности состояний при переходе от монокристалла к тонкой пленке не обнаружено.

Рассчитаны основные оптические функции для тонкой пленки. Обнаруженное отличие от экспериментальных данных незначительное. То есть можем утверждать, что выбранную нами методику расчета можно успешно использовать для прогнозирования оптических и энергетических свойств тонких пленок.

\section{Список литературы}

[1] Romeo N., Bosio A., Tedeschi R., Canevari V. // Mater. Chem. Phys. 2000. V. 66. P. 201. doi 10.1016/S0254-0584(00)003163

[2] Basola B.M., McCandless B. // J. Photon. Energy. 2014. V. 4. P. 040996. doi 10.1117/1.JPE.4.040996

[3] Romeo N., Bosio A., Canevari V., Podestá A. // Sol. Energy. 2014. V. 77. P. 795. doi 10.1016/j.solener.2004.07.011

[4] Paudel N.R., Xiao C., Yan Y. // J. Mater. Sci.: Mater. Electron. 2014. V. 25. P. 1991. doi 10.1007/s10854-014-1834-1

[5] Averin S.V., Kuznetsov P.I., Zhitov V.A., Alkeev N.V., Kotov V.M., Zakharov L.Y., Gladysheva N.B. // Tech. Phys. 2012. V. 57. P. 1514. doi 10.1134/S1063784212110047

[6] Bhattacharya R.N., Contreras M.A., Egaas B., Noufi R.N. // Appl. Phys. Lett. 2006. V. 89. P. 253503. doi 10.1063/1.2410230

[7] Oladeji I.O., Chow L. // Thin Solid Films. 2005. V. 474. P. 77. doi 10.1016/j.tsf.2004.08.114

[8] Mahmood W., Ali J., Zahid I., Thomas A., Haq A. // Optik. 2018. V. 158. P. 1558. doi 10.1016/j.ijleo.2018.01.045

[9] Bosio A., Romeo N., Mazzamuto S., Canevari V. // Prog. Cryst. Growth Charact. Mater. 2006. V. 52. P. 247. doi 10.1016/j.pcrysgrow.2006.09.001

[10] McCandless B.E., Dobson K.D. // Sol. Energy. 2004. V. 77. P. 839. doi 10.1016/j.solener.2004.04.012

[11] Kusnezh V., Petrus R., Il'chuk H., Tuziak O., Zachek I., Rodych V. // J. Nano- and Electronic Physics. 2014. V. 6. P. 020231.

[12] Kraus W., Nolze G. // J. Appl. Crystallogr. 1996. V. 29. P. 301. doi $10.1107 / \mathrm{S} 0021889895014920$

[13] Vanderbilt D. // Phys. Rev. B. 1990. V. 41. P. 7892. doi 10.1103/PhysRevB.41.7892

[14] Perdew J.P., Burke K., Ernzerhof M. // Phys. Rev. Lett. 1996. V. 77. P. 3865. doi 10.1103/PhysRevLett.77.3865

[15] Monkhorst H.J., Pack J.D. // Phys. Rev. B. 1976. V. 13. P. 5188. doi 10.1103/PhysRevB.13.5188

[16] Kashuba A.I., Piasecki M., Bovgyra O.V., Stadnyk V.Yo., Demchenko P., Fedorchuk A., Franiv A.V., Andriyevsky B. // Acta Physica Polonica A. 2018. V. 133. N 1. P. 68. doi 10.12693/APhysPolA.133.68

[17] Schlegel H.B. // J. Comp. Chem. 1982. V. 3. P. 214. doi $10.1002 /$ jcc.540030212

[18] Kohn W., Sham L.J. // Phys. Rev. A. 1965. V. 140. N 4. P. A1133. doi 10.1103/Phys. Rev.140.A1133

[19] Kashuba A.I., Zhydachevskyy Ya.A., Semkiv I.V., Franiv A.V., Kushnir O.S. // Ukr. J. Phys. Opt. 2018. V. 19. P. 1. doi 10.3116/16091833/19/1/1/2018
[20] Kashuba A.I., Franiv A.V., Brezvin R.S., Bovgyra O.V. // Functional Materials. 2017. V. 23. P. 026. doi $10.15407 / \mathrm{fm} 24.01 .026$

[21] Franiv A.V., Kashuba A.I., Bovgyra O.V., Futey O.V. // Ukr. J. Phys. 2017. V. 62. P. 679. doi 10.15407/ujpe62.08.0679

[22] Bloom S., Bergstresser T.K. // Solid State Commun. 1968. V. 6. P. 465. doi 10.1016/0038-1098(68)90056-2

[23] Il'chuk G.A., Kusnezh V.V., Rud' V.Yu., Rud'Yu.V., Shapowal P.Yo., Petrus R.Yu. // Semiconductors. 2010. V. 44. P. 318. doi $10.1134 / \mathrm{S} 1063782610030085$

[24] Il'chuk G.A., Ukrainets V.O., Rud' Yu.V., Kuntyi O.I., Ukrainets N.A., Lukiyanets B.A., Petrus R.Yu. // Techn. Phys. Lett. 2014. V. 30. P. 628. doi 10.1134/1.1792294

[25] Gajdoš M., Hummer K., Kresse G., Furthmüller J., Bechstedt F. // Phys. Rev. B. 2006. V. 73. P. 045112. doi 10.1103/PhysRevB.73.045112 